\title{
African Caribbean hypertensive patients' perceptions and utilization of primary health care services
}

Gina Marie Awoko Higginbottom School of Nursing and Midwifery, University of Sheffield, Sheffield, England, UK

\begin{abstract}
The aim of this article is to illuminate participants' access, utilization and perceptions of primary health care (PHC) services in two cities in England, including factors that militate against or enhance utilization of PHC services for African Caribbean people residing in England. Hypertension remains a major health issue amongst immigrant and UK born African Caribbean people. Current policy agendas are concerned with a reduction in cardiovascular disease and with addressing social exclusion, ameliorating health inequalities, and ensuring that individuals from black and minority ethnic groups receive fair and equitable access, care and treatment. The research conducted in two English cities investigates the meaning and consequences of hypertension and perceptions of PHC services. The research explored the decision making processes that lead to the presentation of individuals in PHC in England for early diagnosis and treatment. This qualitative study draws heavily on the focused ethnography methodological approach. Multiple methods were employed including two focus groups interviews, 21 semi-structured interviews and five vignette-based interviews. Participants numbered 36 in total. Data were analysed with the aid of Atlas/ti. using established principles for analysing ethnographic data. The findings map out the factors influencing early presentation for diagnosis in PHC and perspectives are gained on the quality of the participant/general practitioner (GP) relationship, as perceived by the participants. Participants' satisfaction and dissatisfaction with $\mathrm{PHC}$ services are also evidenced. It is relatively common in English communities of African Caribbean origin to use private PHC services. Motivations for this are evidenced, including historical antecedents. Participants used non-concordance (ie, non-compliance with prescribed medications) and traditional Caribbean herbal remedies as strategies of empowerment.
\end{abstract}

Key words: chronic illness; ethnicity; hypertension; primary health care

Received: November 2004; accepted: August 2005

\section{Introduction}

This article presents selected findings from a focused ethnographic study of hypertension in people of African Caribbean origin residing in

Address for correspondence: Gina Marie Awoko Higginbottom, School of Nursing and Midwifery, University of Sheffield, Herries Road, Sheffield S5 7AU, UK.

Email: g.higginbottom@sheffield.ac.uk
England. The research investigates the meaning and consequences of hypertension from the participants' perspectives exploring both personal and societal influences on the development and management of hypertension, health beliefs, risk perception and decision making processes. In this paper specific findings are presented regarding participants' perceptions and utilization of primary health care (PHC) services in England. The aim of this paper is to illuminate participants' access, utilization and perceptions of PHC services in 
England, including factors that militate against or enhance utilization of PHC services for African Caribbean people residing in England.

\section{Background}

\section{African Caribbean people in England}

Communities of African Caribbean origin, who have migrated to England, have ancestry in the Caribbean Islands, including Barbados, Jamaica, Trinidad and Tobago, the Windward Islands, the Leeward Islands, Guadeloupe and the Bahamas, which were former colonial states.

\section{Clarifying terminology}

The term 'African Caribbean' is adopted to describe participants in this study who have origins in the Caribbean and connections to Africa via historical antecedents. Significantly, communities of Caribbean origin in England often adopt the term African Caribbean to describe community groups and associations. In this sense, the term reflects the group acceptance of the term as existing within a social reality, and such usage demonstrates that the term has meaning and validity for the group (Modood et al., 2002). Adoption of this term does not deny the huge variation in language, culture and traditions within the Caribbean Islands; this is acknowledged. The research also employed the principle of self-assignation of ethnicity (Nazroo, 1997) by all participants, notwithstanding the fact that self-assignation alone does not necessarily confer membership of a group (Modood et al., 2002). Rather, membership of the group is both an individual subjective view and subject to conferment by at least some members of that group. However, observers have noted the fluid and situational nature of ethnicity (Jenkins, 1997; Atkin et al., 2002) depending on the context, time and place.

There exists considerable heterogeneity in communities in England that might be described broadly as African Caribbean, and therefore a myriad of variables exist that might impact on the health experience, health beliefs and health actions of this community. This diversity is well documented (Alexander, 1999; Elam et al., 2000) and includes such issues as motivation for migration, language, religion, socio-economic class, level of education, dialect spoken, type of employment, family composition and social networks. Commonality does exist in that a large number of the UK's African Caribbean community live in the major conurbations (Dorsett, 1998), although not exclusively so. In respect of the provision of PHC for African Caribbean people in England, insight by PHC professionals into the ethno-history of patients and clients is desirable for effective communications and therapeutic encounters (Morgan, 1995; Gerrish, 1999; 2000).

\section{Ethnicity and the experience of hypertension as a chronic illness}

People from ethnic minority groups in England tend on the whole to experience greater ill-health than the general population, and this inequality has been documented in a number of studies over past decades (Marmot et al., 1984; Donovan, 1985; Thorogood, 1988; Health Education Authority, 1994; Smaje, 1995). For example, in relation to hypertension, Nazroo (1997) states that Caribbean participants in the Fourth National Survey of Britain's Ethnic Minorities experienced almost $50 \%$ higher rates than whites. However, the data did demonstrate a much less marked gender variation for men, as for Caribbean women the rate was almost $80 \%$ higher than in the white population. Other measures of morbidity and mortality in this study illustrate similar inequalities for ethnic minority communities in England. The 1999 Health Survey for England (Department of Health, 2000) further confirms the experience of poor health for ethnic minority populations more accurately by providing evidence of diagnosis, measured hypertension and treatment rates. The study demonstrated an increased prevalence of hypertension for women of Black Caribbean, Indian, and Pakistani origin. Bangladeshi and Irish women exhibited lower rates of hypertension then the general population. In this study Pakistani, Bangladeshi and Chinese men demonstrated lower rates of hypertension then men in the general population. Irish, Bangladeshi and Black Caribbean men were more likely to have received therapy for hypertension than men in the general population. For all ethnicities the experience of morbidity was correlated to socioeconomic group, in that greater morbidity and mortality was experienced in lower socio-economic groupings. 
Current policy agendas are concerned with a reduction in cardiovascular disease (Department of Health, 1999) and with addressing social exclusion, ameliorating health inequalities, and ensuring that individuals from black and minority ethnic groups receive fair and equitable access, care and treatment (Department of Health, 1999). Chronic illness experience such as hypertension is mediated by social class, gender and age. The steep social class gradients that are associated with increased morbidity and mortality in the general population have been recognized for several decades (Townsend and Davidson, 1982). Although ethnic minority communities experience greater ill-health then the general population (Nazroo, 1997), this experience is inextricably linked to social class dimensions (Nazroo, 1998; Karlsen and Nazroo, 2002a, b; Nazroo, 2003).

\section{The meaning and consequences of hypertension}

\section{Historical antecedents}

Torkington (1995) claims that for African Caribbean communities in Europe today, the historical influence of the transatlantic slave trade, colonialism and imperialism continue to exert influence. This is manifested in the present economic and social experience of African Caribbean communities in the UK. Leininger (1991, p. 48) terms this type of retrospective exploration 'ethno-history' and defines ethno-history as:

Those past facts, events, instances, experiences of individuals, groups, cultures and institutions that are primarily people-centred (ethno) and which describe, explain and interpret human life ways within particular cultural contexts and over short or long period of times.

Moreover, it is essential that health care professionals develop an understanding of the ethno-history of patients and clients, in order to provide culturally congruent care and to challenge ethnocentrism in health care. In this study detailed biographical information is elicited from participants regarding, length of migration, birthplace of parents and grandparents, religious affiliations and so on.

The experience of diagnosis of hypertension is very much mediated by the prevailing social, economic and cultural influences in an individual's life (Van Wissen et al., 1998). It may be difficult for individual patients to separate out components of their life experience, such as illness or disease, from an overall perspective on their 'quality of life' (Van Wissen et al., 1998). Therefore, an affluent employed person with few stressors is likely to respond to the diagnosis of hypertension in a very different way to an individual who may be unemployed and have multiple life stressors, and be living in impoverished circumstances. Indeed the 'biographical disruption' which is a result of chronic illness (Bury, 1982) is said to be less frequently encountered by those in privileged positions (Williams, 2000). It is possible that different personality types may respond in different ways, depending on the individual's ability to adapt to changed life circumstances. Furthermore, the level of social support that individuals experience may also be a factor that impacts on perceptions regarding hypertension.

Concepts of health and illness are also culturally defined (Helman, 2001). Therefore, as might be expected, the experience of hypertension and interpretations of the meaning and consequences of hypertension are uniquely individual. Van Wissen et al. (1998) conducted a qualitative study using interviews with a population in New Zealand to explore the experience of living with high blood pressure. The majority of the participants were of European origin, with only two patients of Maori origin, and this may limit the significance of the findings to an African Caribbean population in England. However, some pertinent insights are illuminated. Emergent themes in the findings focused on the participant's reconstruction of a personal outlook on life and the associated health beliefs and behaviours, such as meeting the commitments of daily living outside of the family, recognizing symptoms, reactions to diagnosis, modification of lifestyle and medication.

The findings in Van Wissen's study highlight the individual's life experience as mediating the experience of high blood pressure. The patient therefore is viewing the diagnosis and experience of hypertension through the lenses of their total life experience, whereas the health professional may view the patient as an individual with a diagnosis of hypertension as that individual presents in surgery or clinic. This compartmentalization or construction of illness and disease as a discrete entity is characteristic of a Western biomedical approach, but 
does not necessarily reflect lay perspectives that may embrace a more holistic perspective. Furthermore, the social, cultural, and economic context in which a chronic disease such as hypertension is experienced is not static, but changing and dynamic (Van Wissen, 1998). This study suggests that health professionals in the prescription and monitoring of anti-hypertensive therapies do not take account of this dynamic experience, and therefore patients empower themselves by modifying treatment programmes by non-adherence in order to meet their lifestyles.

\section{Access to PHC}

PHC services are the first level of services available to the general public, usually, although not exclusively, located within GPs' surgeries and health centers (some individuals, especially transient communities, may use Accident and Emergency Departments as a form of PHC). More recently, newer forms of PHC have emerged such as walk-in centres, sexual health shops and mobile health buses. A range of practitioners, including doctors, community nurses, midwives and health visitors, may deliver PHC.

The research evidence reviewed on access to PHC by ethnic minority communities can be grouped into emergent key themes:

1) The barriers and obstacles encountered during access to PHC (Chaturvedi et al., 1997; Smaje and Le Grand,1997; Smaje, 1998; Free et al.,1999; Farooqi et al., 2000).

2) Language barriers between clients and health professionals create obstacles in obtaining relevant and appropriate PHC (Chaturvedi et al., 1997; Modood et al., 1997; Nazroo, 1997; Carr-Hill, 1996; Smaje, 1998; Pui-Ling et al., 1999).

3) Inequalities in care pathways and referrals to secondary care services. A number of studies included a comparative dimension with the indigenous white population (Commander et al., 1997; Modood et al., 1997; Nazroo, 1997; Smaje and Le Grand, 1997; Smaje, 1998; Commander et al., 1999). This enabled the researchers to establish the existence of inequality in relation to access to PHC by minority ethnic communities.

4) Other key issues relate to point 3 and give rise to inequalities such as discrimination/racism, cultural issues/clash, practitioner's knowledge and patient knowledge of services.
No studies focused exclusively on African Caribbean populations, but such studies are urgently needed, given the greater experience of ill-health and prevalence of chronic diseases such as hypertension.

\section{The study}

The aim of this article is to illuminate participants' access, utilization and perceptions of PHC services in England, including factors that militate against or enhance utilization of PHC services for African Caribbean people residing in England.

\section{Methodology}

Participants' experiences and strategies in relation to hypertension are examined from an ethnographic perspective (Spradley, 1979; Hammersley and Atkinson, 1995; Fetterman, 1998). It is acknowledged that a classical or pure ethnography demands the inclusion of participant observation as an important method of data collection. However, a number of published studies exist that draw on ethnographic tradition but do not necessarily use participant observation (Van Maanen, 1995; Gillibrand and Flynn, 2001). Within nursing research, especially in North America, a new genre of ethnography has gained prominency; that of focused ethnography (Muecke, 1994). Morse (1994) and others make distinctions between focused ethnography and the traditional anthropologic ethnography. Characteristics of focused ethnographies are:

- conceptual orientation of a single researcher

- the focus on a discrete community or organization or social phenomena

- problem focused and context specific

- a limited number of participants

- participants usually hold specific knowledge

- episodic participation observation

- conducted for development in health services.

(After Muecke, 1994)

The focused ethnographic approach has been used specifically to explore symptom recognition, diagnosis, perceptions of illness (Hudelson et al., 1995), and all features that appear in this research, which is why this methodological approach was 
selected. Gillibrand and Flynn (2001) term their study 'adjectival ethnography', a term that recognizes that the methodology used is not a classic ethnographic study, but primarily uses interviews as data collection. The term selected to describe this study is ' $a$ focused ethnography which draws on the adjectival tradition'. This is a synthesis of two approaches described earlier adjectival ethnography (Van Maanen, 1995; Gillibrand and Flynn, 2001), where ethnography primarily relies on interview data and that which Morse (1994) describes as a focused ethnography. The research design also included a consumer research advisory group, consisting of six key community members and individuals who had experienced hypertension. The group may also be regarded as key informants (Hammersley and Atkinson, 1995).

\section{Reflexivity}

Reflexivity is an important dimension of ethnography. Murphy et al. (1998, p. 188) define reflexivity as:

... we mean sensitivity to the ways in which the researcher's presence in the research setting has contributed to the data collected and their own a priori assumptions have shaped the data analysis.

In this study reflexivity was achieved via a narrative journal which included a self-reflection and critical deconstruction of my own ethnic identity and the influence of this on the research process.

\section{Sample}

Participants in this study were African Caribbean people with hypertension aged between 37 and 82 years (median age 59.5 years). The sample comprised almost equally of male and female participants. Several participants had migrated as young people or children to England; two were born in England, with around two-thirds migrating during the 1950/1960s. Of the later group residency in England ranged from 20-47 years with most residing in England for over 40 years. The sample was generated by contacting 27 general practice surgeries in two cities with high populations of African Caribbean patients. Participants were recruited via 13 general practices. In order to achieve maximum phenomena variation ie, ensuring many different variations of the data in a given sample are explored (Higginbottom, 2004) recruitment via a snowballing technique was used (refers to a technique whereby additional participants are recruited via existing participants). It is a particularly useful strategy for hard to reach groups (Elam and Fenton, 2003) and further recruitment occurred via faith and religious groups, community groups and associations generating 36 participants in total (30 participants were recruited via GPs and six via other methods). For inclusion in the study participants were required to be adults of African Caribbean origin and have been diagnosed with hypertension, no age limit either upper or lower was imposed.

\section{Data collection}

The process of data collection and links between the three data collection tools

The data were collected during 2002-2003. This research employed three discrete but related phases of data collection. Each data collection stage builds on the previous analysis and findings, enabling a deeper investigation into aspects of the experience of African Caribbean people with hypertension. In ethnographic research this process is known as progressive focusing (Silverman, 2000). The methods used are focus group interviews (FGI), semi-structured interviews and vignette interviews. The FGI established the broader issues of investigation in relation to the research questions. A pilot FGI and one in each of the study locations were conducted, three in total. The findings from the FGI influenced the development of the topic guide for semi-structured interviews that forms the main method of data collection. All data collection was tape recorded and transcribed verbatim. Reflective field notes were also recorded. In this paper selected findings, perceptions of PHC, are presented from the semi-structured interviews considered embracing four themes:

1) running to the doctor

2) satisfaction/dissatisfaction with $\mathrm{PHC}$ services

3) non-concordance

4) motivations for use of private GPs.

Achieving rigour in qualitative research

Rigour and robustness in qualitative research is to some extent established via a self-conscious and 
reflective approach, accompanied by an explicit methodological framework. In order to establish the rigour and scientific value of this research, the work conducted was considered in the light of the framework for assessing the quality of qualitative research postulated by Guba and Lincoln (1989):

- Confirmability: Achieved in this research via self-conscious reflective approach, the general methods and procedures are described in detail ensuring a transparent audit trail.

- Dependability: Demonstrated in this study by the adoption of a theoretical methodological approach and the consistency and stability of the research and methods, supervised by experts in the field as the study formed doctoral research.

- Transferability: Refers to typicality in relation to other participants in similar locations. Therefore, it may be possible to draw empirical generalizations. Empirical generalizations are dependent on the achievement of maximum phenomena variation as is the case in this study. For example in this study it was necessary to include both first and second-generation migrants, as well as ensuring a gender and age mix. In addition both economically active and retired people are included in the study, thus ensuring a heterogeneous sample of African Caribbean people with hypertension.

- Credibility: The focus of concern in this dimension is authenticity and accuracy.

- Reliability: In this study this is further enhanced by the validation of emergent findings and conclusions by the consumer research advisory group. Patient and public participation in research in the $\mathrm{UK}$ is viewed as a principle of good research practice. The group and researcher met regularly during the progression of the research. Additionally participants in the study were provided with the opportunity to comment on the findings.

\section{Ethical considerations}

Ethical permissions were obtained from two Local Research Ethics committees and the anonymity and confidentially of all participants maintained. Informed consent was obtained form all participants. In reporting the findings in this paper all participants are given a pseudonym.

\section{Data analysis}

Data were analysed by the author using Roper and Shapira (2000) framework for the analysis of ethnographic data with the aid of Atlas/ti. qualitative data analysis software. (The software package does not analyse the data, interpretation, deconstruction and reconstruction of the data, synthesis and abstraction is dependent on the cognitive skills of the researcher.) Roper and Shapira's (2000) framework for analysis of ethnographic data was selected because of:

- the relevance to ethnographic data

- the clarity and transparency of the approach

- the systematic approach to analysis, following clearly defined steps

- the relevance of the approach to pragmatic health service research

- the compatibility of the approach with computer assisted qualitative data analysis such as Atlas/ti.

\section{Steps in analysis}

The process of qualitative data analysis is characterized by identification and classification of data and progresses to abstract generalizations, explaining patterns of behaviour within the cultural group. The process described below is not linear, but undulating and convoluted. This characterizes the iterative process (Silverman, 2000) associated with qualitative research as preliminary interpretations are challenged and data is revisited in the light of further data collections and new insights into the data. Analytical induction is the sine qua non of ethnographic.

Steps in analysis (after Roper and Shapira, 2000):

- coding for descriptive labels

- sorting for patterns

- identification of outliers or negative case

- generalizing: constructs and theories

- memoing: reflective remark.

\section{Findings}

The findings presented illuminate participants' perspectives on utilization and perceptions of PHC services. In presenting verbatim comments to illuminate the findings it is important for the reader to note that African Caribbean dialect or 'patois' is typified by a unique syntax, morphology and 
grammatical expression (Scott, 1998) which is derived from both West African (as a result of the transatlantic slave trade) and English, French or Spanish languages (dependent on the particular colonial power in specific Caribbean Islands).

\section{'Running to the doctor'}

The reasons why African Caribbean people may be reluctant to present for early diagnosis or use a GP are not established in the literature; but they are documented in this research, which enhances understanding of why African Caribbean people may be reluctant to use GPs and present for early diagnosis.

Participants in the study presented some evidence to suggest that seeking professional medical attention, at the onset of a dysfunction or disease, may not be a cultural norm for individuals who have migrated to England from the Caribbean. This is evidenced in the data, as historical antecedents, prior to migration. In past decades in the Caribbean, the lack of a state-provided formal PHC service meant that through necessity, communities had to develop self-care systems that appear to be deeply embedded in the culture and traditions of the participants. Moreover, medical provision that did exist was only available to the wealthy that could afford such medical care. Loretta commented:

Something can be wrong, could be serious and it take ages for them [African Caribbean people], for it to be diagnosed because they're not running to the doctor ... not keen on running to the doctor. That's the sort of way they would feel. Running to the doctor.

\section{(Interview 17)}

Loretta appears to view presenting oneself at the GP surgery as soon as symptoms become evident negatively. The notion of 'running to the doctor' is used by Loretta as a pejorative concept. Hyacinth and Loretta commented on trends they had observed in England and Jamaica in relation to younger African Caribbean people and their readiness to use PHC services. Participants viewed resourcefulness and self-care, as an essential component of life in the Caribbean. This may mitigate against early presentation for diagnosis in PHC of conditions such as hypertension. Clifford reiterates this point:

You only hear about doctors. You never see one, 'cause they, well, it's mostly people living in the country, there are bush things [herbal remedies]. You know, like, they drink bush tea, they got bush this, bush that.

(Interview 8)

Delbert elucidates further on the scarcity of doctors and PHC in past decades in the Caribbean. Both Clifford and Delbert emphasized how the lack of access to formal PHC services may have contributed to patterns of self-care that existed, and reliance on traditional Caribbean herbal remedies:

$\ldots$ in those days when we were in the Caribbean, if someone said they had a fever they didn't go to the doctor, they just boil some bitter herbs and drink it. Each year your knowledge increases so we don't know what's out there now ... The only time you heard about a doctor was when you heard one was going to operate in Montego Bay and we always call the doctor 'butcher man', 'butcher people'. I have never been to a doctor in Jamaica.

(Interview 9)

Delbert also seems to imply that he did not hold the doctor who was available in Montego Bay in very high esteem.

\section{Satisfaction/dissatisfaction with PHC services}

Participants in this study, largely expressed satisfaction with the PHC services they had received in respect of their high blood pressure, and were very keen to emphasize their positive regard, especially for their GP. In this study all the participants regularly consulted with their GP rather than the practice nurse. This I found quite surprising given the role practice nurses have in chronic disease management in PHC. Carmel, Loretta, Wesley and Delbert, had seen the practice nurse occasionally, for blood pressure monitoring and blood tests. Carlene explains why she values her present GP:

She'll sit down and she'll talk to you. That's why now, I've started seeing her.

Because she's got that nice smile. 
You know, it's genuine and she can spend time talking to use, you know, even your upset she'll go 'well, you need to go Carlene. You need to get it sorted'

She's completely different.

(Interview 15)

Carlene appears to focus very much on the nonverbal communication her GP displays during consultation such as her smile, genuineness and the feeling that she has more time for her patients. Having time available for consultations with patients, seemed to be key in relation to participants' expressed satisfaction with their GP.

\section{Non-concordance}

Individuals who experience chronic illnesses, such as hypertension, diabetes or arthritis, may be non-concordant with medication (Benson and Britten, 2002). This is not specific to African Caribbean's, but also a well-documented feature of individuals who are members of the general population in England.

Participants gave me a range of explanations for their non-concordance with prescribed medication. Some participants expressed anxiety about potential and actual side-effects from the medication. This information was gleaned either from the information leaflet in the medication box, from their GP or as a result of side-effects they had experienced. Hyacinth explains:

... I said to the doctor, because I was having all the side-effects and I'm aware that it could affect your heart as well, and bearing in mind that my Mum died of heart attack, I thought there is no way, I mean heart problem, there's no way I'm going to take some tablets that would actually, you know, put my heart at risk, you see.

\section{(Interview 19)}

Hyacinth was also of the view that once a tablet had entered her body she would have no control over which organs the medication might affect, as she commented:

I just don't like it. I feel as though, if there's something wrong, I've got blood pressure, and that tablet could create more problems and just put pressure, because once you put a tablet in your body, that's my thinking anyway, it, you often read this little, you know, leaflet that they put inside and you could affect your kidney and it could affect your heart and I thought then, if I'm only going to have one problem, which is blood pressure, and end up with, with heart problem, or kidney failure or whatever goes it, I'm better off looking at alternative medicine, if it can help.

(Interview 19)

It seems that the drug information leaflet, far from empowering Carmel and Hyacinth with more information, actually fuelled their anxiety and in many senses the information provided was counterproductive to them taking the prescribed medication. Other participants such as Petra, Carlene, Delbert, Carlton, Loretta and Hyacinth had experienced side-effects such as dizziness, headaches, and oedema of the legs and impotence this had pre-empted their non-concordance.

\section{Motivations for use of private GPs}

A tradition of using private PHC and GPs, exists in the Caribbean for those people who could afford to, and lived in close enough proximity (usually towns) to access the services (Morgan, 1993). Most people in the study were aware of the existence of private GPs in England through family, friends or relatives who were using the services. It seems that the use of these services also served a social function, as a coach or bus would be hired to take a number of people to different cities in order to use such services.

The study locations were Sheffield and Nottingham, although participants mentioned several cities where they had accessed private medical services, eg, Derby, Birmingham, Leicester. I am uncertain as to what extent this might be a deliberate strategy to avoid recognition by the participants' own NHS GP of this activity, although, some participants had visited private GPs in their place of residence, eg, Sheffield, Nottingham and Wolverhampton. As Barbette comment:

I get lots of invitations from people who are going out to Leicester to private Dr's.

(Interview 14)

The social nature of such occasions is emphasized by the notion of receiving an invitation from a 
friend as in a party or wedding. Knowledge of the existence of private GPs in other towns also demonstrates the tacit knowledge held within communities and the power of 'word of mouth' as to the best of my knowledge private GPs do not advertise their services.

The use of private medical practitioners also appears to be influenced by the use of private medical care by affluent and wealthy people in England, leading some participants to conclude that private $\mathrm{PHC}$ accessed is superior to that which is received in the NHS. Carlton remarks:

I think they think they get what they, if you pay for something you get what you pay for. Erm, sometimes, when I'm in the doctors, they feel that they haven't, there's something about having, when you pay for something, you're getting the best. You know. Because it's free on the National Health Service, you're not getting the best, where as the rich people, they pay for it and they get the best.

(Interview 21)

Carlene and Delbert both believed that the actual treatments or medication received were of a superior quality to medications available on the NHS. Not all participants in this study expressed enthusiasm about the use of private GPs. Some participants were sceptical about differences in quality of NHS medications and privately prescribed medication.

\section{Discussion}

The findings presented form a part of a larger ethnographic study, in this sense the generalisability of the selected findings presented may be open to question, however important insights are gained in an area to date which has been neglected. Participants in this study most commonly expressed satisfaction with the PHC services received, which were mainly consultations with GPs. This was surprising given the role of practice nurse in chronic disease management, however it could be related to the perception that African Caribbean people with hypertension present management challenges (Morgan, 1993), although in respect of non-concordance African Caribbean people share commonality with the general population (Benson and Britten, 2002). Within the consultations time for discussion, active listening by the PHC professionals, and positive non-verbal communication were aspects of consultations that were considered to be important and valued by the participants. The lack of openness in communication between participants and health professionals during consultation was also a key finding, especially in relation to potential side-effects. Although, not all participants wished for frank discussion about their conditions. Furthermore, aside from communication directly focused on hypertension, participants also demonstrated a lack of openness during consultations, choosing not to share aspects of their everyday lives. Thus it may be concluded that greater openness during communication between participants and health professionals, may have resulted in a greater willingness by the participants in this study to discuss side-effects and prevent cessation of medication which could potentially have dangerous health consequences. In addition, greater openness may have facilitated disclosure of the use of herbal remedies.

Some participants used private GPs which may reflect a tradition of paying for medical services in the Caribbean or dissatisfaction with PHC in the UK. Those participants who did use private GPs did so simultaneously with NHS GP consultations. Benefits from the participants' perspectives were said to be a greater explanation of the condition, and superior quality medications, to that available from the NHS. The use of private GPs by some participants in this study may signify dissatisfaction with NHS provision. There also existed amongst some participants scepticism regarding the value of private GPs.

Where dissatisfaction had existed with current NHS GP and PHC services, participants appeared to actively manage the situation by changing their GP. Most of the participants were not familiar with new forms of PHC services such as 'walk-in centres'.

Within the domain of non-concordance, openness in communications between the patient and GP emerged as an important consideration. Some participants did share with their GP their nonconcordance and use of herbal remedies, but most did not, and this might have serious consequences for the health of the individual concerned. However, it was clear that reciprocity was not achieved in many GP/patient relationships, as openness was also sometimes lacking on the part of the GP.

In considering participants' concordance and non-concordance with medication, the data in this 
study suggests some important future research questions: for example, what are the processes by which participants estimate risk to their health? For example, from the perspective of those participants who had ceased taking medication because of potential rather than actual side-effects, what were the decision making processes that enabled participants to balance this risk against the potential risk of not taking medication? There may also be a synergistic relationship between self-care and use of herbal remedies that would warrant further investigation.

\section{Conclusions}

The findings from this study present important evidence that addresses the paucity of research in this area. Realizing the NHS modernization programme (Department of Health, 1997) in a PHC led NHS in England (Department of Health, 1999) requires insight into how and why inequalities in access and use of PHC services exist for different population groups. This study serves to address the knowledge gap in this domain, and important insights are gained for all PHC practitioners including practice nurses and district nurses.

Most participants in this study expressed satisfaction with PHC services received. However, the findings of this study do indicate that a lack of openness does exist betweens some PHC professionals and African Caribbean people with hypertension. Reflecting on the findings of this study, key issues are the need for good communication in relation to early diagnosis, ongoing care and chronic disease management; strategies the patients might use to self-manage their condition, such as herbal remedies and use of private GPs, and the wider context of the individual's life and how this might impact upon their health status. However, it is worth noting that many of the wider structural issues that the participants identified as either causing or impacting upon their hypertension may be outside the remit of most PHC professionals.

First, I suggest two approaches to enhance management in PHC practice. I would recommend that in order to engage more closely with all patients, including African Caribbean patients with hypertension, PHC professionals as a fundamental starting point elicit the individual patients' explanatory model of health and illness. This would enable many of key issues identified in the findings presented to be made explicit and addressed, such as concordance, use of herbal remedies, use of private GPs, satisfaction or otherwise with treatment. A number of models exist to guide practitioners in eliciting the explanatory model (EMs) of clients and patients (Kleinman, 1980; Weinmann et al., 1996; Weis, 1997; Lloyd et al., 1998). I would recommend that key constructs be integrated into the preliminary nursing assessments of African Caribbean people with hypertension in PHC. Kleinman's seminal work focuses on eight questions to uncover a patient's or client's EM, and these might allow cultural adaptation of practice within PHC services, by establishing the ground for negotiation between patient and practitioner. The questions follow:

1) What do you call the problem?

2) What do you think has caused the problem?

3) Why do you think it started when it did?

4) What do you think the sickness does? How does it work?

5) How severe is the sickness? Will it have a short or long course.

6) What kind of treatment do you think the patient should receive? What are the most important results you hope to achieve from this treatment?

7) What are the chief problems the sickness has caused?

8) What do you fear about the sickness?

Within the context of contemporary PHC the eight questions listed may be considered over long, in which case consideration should be given to the shorter models such as Weinmann et al. (1996), Lloyd et al. (1998) and Weiss (1997). The complexity of patients' EMs means that concordance with therapies is unlikely to be achieved unless the PHC professional is aware of the dissonance between practitioner and patient EMs.

\section{Acknowledgements}

This study was funded via the NHS Executive via a National Primary Care Research Fellowship. I would like to acknowledge my $\mathrm{PhD}$ supervisors Professors N. Mathers, Kate Gerrish and James Nazroo, and also the members of Consumer 
Research Advisory Group whose insights were invaluable.

\section{References}

Alexander, Z. 1999: The Department of Health: study of Black, Asian and Ethnic Minority Issues. London: The Stationary Office.

Atkin, K., Ahmad, W.I.U. and Jones, L. 2002: Young South Asian deaf people and their families: negotiating relationships and identities. Sociology of Health and Illness 24, 21-45.

Benson, J. and Britten, N. 2002: Patients' decision about whether or not to take antihypertensive drugs: a qualitative study. British Medical Journal 325, 873-76.

Bury, M. 1982: Chronic illness as disruption. Sociology of Health and Illness 4, 167-82.

Carr-Hill, R.A., Rice, N. and Martin, R. 1996: Socio-economic determinants of rates of consultation in general practice based on the fourth national morbidity survey of general practices. British Medical Journal 312, 1008-12.

Chaturvedi, H.R. and Ben-Shiomo, Y. 1997: Lay diagnosis and health-care seeking behaviours. The Lancet 350, 1578-83.

Commander, M.J., Sashidharan, S.P., Odell, S.M. and Surtees, P.G. 1997: Access to mental health care in an inner city Health District 11: association with demographic factors. British Journal of Psychiatrists 170, 317-20.

Commander, M.J., Odell, S.M., Williams, K.J. and Surtees, P.G. 1999: Pathways to care for alcohol use disorders. Journal of Public Health Medicine 21, 65-69.

Department of Health. 1997: The new N.H.S. - modern and dependable (Cm3807). London: The Stationary Office.

Department of Health. 1999: Saving lives - our healthier nation (Cm4386). London: The Stationary Office.

Department of Health. 2000: The Health Survey for England 1999. London: Stationary Office.

Donovan, J.L. 1985: Black people's health: a study of the perceptions and experiences of health and healthcare of black people in London. Unpublished PhD thesis. Queen Mary and Westfield College, London. 35-5712.

Dorsett, R. 1998: Ethnic minorities in the inner city, Bristol: The Policy Press and the Joseph Rowntree Foundation.

Elam, G. and Chinouya, M., with the Joint Health Surveys Unit. 2000: Feasibility Study for Health Surveys among Black Populations Living the UK: stage 2 - diversity among Black African Communities. London: The Stationary Office.

Elam, G. and Fenton, K.A. 2003: Researching sensitive issues and ethnicity: lessons from sexual health. Ethnicity \& Health 8, 15-27.

Farooqi, A., Nagra, D., Edgar, T. and Khunti, K. 2000: Attitudes to lifestyle risk factors for coronary heart disease amongst South Asians in Leicester: a focus group study. Family Practice 17, 293-97.
Fetterman, D.M. 1998: Ethnography: step-by-step. Thousand Oaks, CA: Sage Publications.

Free, C., White, P., Shipman, C. and Dale, J. 1999: Access to and use of out-of-hours services by members of Vietnamese community groups in South London: a focus group study. Family Practice 16, 389-74.

Gerrish, K. 1999: Inequalities in service provision: an examination of institutional influences on the provision of district nursing care to minority ethnic communities. Journal of Advanced Nursing 30, 1263-71.

Gerrish, K. 2000: Individualized care: it's conceptualization and practice within a multiethnic society. Journal of Advanced Nursing 32, 91-99.

Gillibrand, W. and Flynn, M. 2001: Forced externalization of control in people with diabetes: a qualitative exploratory study. Journal of Advanced Nursing 34, 501-10.

Guba, E. and Lincoln, Y. 1989: Fourth Generation Research. Thousand Oaks, CA: Sage.

Hammersley, M. and Atkinson, P. 1995 Ethnography principles and practice. London: Routledge.

Health Education Authority. 1994: Black and minority ethnic groups in England. London: Health Education Authority.

Helman, C. 2001: Culture, health and illness, fourth edition. Oxford: Butterworth-Heinemann.

Higginbottom, G.M.A. 2004: Sampling issues in qualitative research. Nurse Researcher 12,7-19.

Hudelson, P., Huanca, T., Charaly, D. and Cirpa, V. 1995: Ethnographic studies of ARI in Bolivia and their use by the National ARI Programme. Social Science and Medicine 41, 1677-83.

Jenkins, R. 1997: Rethinking ethnicity. London: Sage Publications.

Karlsen, S. and Nazroo, J.Y. 2002a: Agency and structure: the impact of ethnic identity and racism on the health of ethnic minority people. Sociology of Health and Illness 24, $1-20$.

Karlsen, S. and Nazroo, J.Y. 2002b: Relation between racial discrimination, social class and health among ethnic minority groups. American Journal of Public health 92, 624-31.

Kleinman, A. 1988: The illness narratives: suffering, healing \& the human condition. New York: Basic books.

Leininger, M. 1991: Culture, care diversity and universality: a theory of nursing. New York: National League for Nursing.

Marmot, M.G., Adelstein, A.M., Bulusu, L. and OPCS 1984: Immgirant mortality in England and Wales 1970-78: causes of death by country of birth. London: HMSO.

Modood, T., Berthoud, R., Lakey, K., Nazroo, J., Smith, P., Virdee, S. and Beishon, S. 1997: Ethnic minorities in Britain: diversity and disadvantage. London: Policy Studies Institute.

Modood, T., Berthoud, R. and Nazroo, J. 2002: 'Race', Racism and Ethnicity: a response to Ken Smith. Sociology 36, 419-27.

Morgan, M.A. 1993: Beliefs and responses to hypertension: patients and practitioners' perspectives. Unpublished $\mathrm{PhD}$ thesis. United Medical and Dental School, 44-11356.

Morgan, M.A. 1995: The significance of ethnicity for health promotion: patients' use of antihypertensive drugs in inner London. International Journal of Epidemiology 24, S79-S84. 
Muecke, M.A. 1994: On the evaluation of ethnographies. In Morse, J.M. Editor, Critical issues in qualitative research methods. Thousand Oak, CA: Sage.

Murphy, E., Dingwall, R., Greatbatch, D., Parker, S. and Watson, P. 1998: Qualitative research methods in health technology assessment: a review of the literature. Health Technology Assessment 2, 16.

Morse, J.M., editor, 1994: Critical issues in qualitative research methods. Thousand Oaks: Sage Publications, 187-209.

Nazroo, J.Y. 1997: The health of Britain's ethnic minorities. London: Policy Studies Institute.

Nazroo, J.Y. 1998: Genetic, cultural or socioeconomic vulnerability? Expanding ethnic inequalities in health. Sociology of health and illness 20, 277-84.

Nazroo, J.Y. 2003: The structuring of ethnic inequalities in health: economic position, racial distribution and racism. American Journal of Public Health 93, 277-84.

Pui-Ling, Li., Logan, S., Yee. L. and Ng, S. 1999: Barriers to meeting the mental health needs of the Chinese community. Journal of Public Health Medicine 21, 74-80.

Roper, J.M. and Shapira, J. 2000: Ethnography in Nursing Research. Thousand Oaks, CA: Sage.

Scott, P. 1998: Lay beliefs and the management of disease amongst West Indians with diabetes. Health and Social Care in the Community 6, 407-19.
Silverman, D. 2000: Doing qualitative research: a practical handbook. London: Sage.

Smaje, C. 1995: Health, 'race' and ethnicity: making sense of the evidence. London: Kings Fund.

Smaje, C. 1998: Health, 'race' and ethnicity: making sense of the evidence. London: Kings Fund.

Smaje, C. and Le Grand, J. 1997: Ethnicity and the use of health services in the British NHS. Social Science and Medicine 45, 485-96.

Spradley, J.P. 1979: The ethnographic interview. Fort Worth: Harcourt Brace Jovanich College Publishers.

Thorogood, N. 1988: Health and the management of daily life amongst women of Caribbean origin living in Hackney. Unpublished PhD thesis. London: Goldsmith College.

Torkington, N.P.K. 1995: Black migrant women and health. Women's Studies International Forum 18, 153-58.

Townsend, P. and Davidson, N. 1982: Inequalities in health (the Black Report). Middlesex: Penguin.

Van Maanen, J. 1995: Representation in ethnography. Thousand Oak, CA: Sage.

Van Wissen, K., Litchfield, M. and Maling, T. 1998: Living with high blood pressure. Journal of Advanced Nursing 27, 567-74. 